\title{
Sparks fly as electrostatic facility fizzles out
}

Declan Butler, Paris

European nuclear physicists are up in arms over a decision to shut one of the world's largest van de Graaff electrostatic accelerators, the two-million-volt Vivitron in Strasbourg, France.

The researchers say that the move is premature, as the field's next generation of machines is still on the drawing board. They fear that the measure will drive some Europeans who study 'exotic' nuclei out of the field for good.

The study of exotic nuclei - which have an unstable balance of neutrons and protons, and so are prone to rapid radioactive decay - is one of the hottest areas of contemporary nuclear physics. The research is expected to yield clues as to how elements form in stars and supernovae. It could help to refine the standard model, and may even give rise to better medical isotopes.

The Vivitron is one of three facilities in Europe that generate beams of stable ions, alongside machines at Legnaro in Italy and Jyväskylä in Finland. But a more recent technology uses beams of radioactive ions, and there is a consensus in the United States, Europe and Japan that building next-generation machines that use this approach is the top priority for studying exotic nuclei.

The plan to close the Vivitron was announced by the National Institute of Nuclear and Particle Physics, part of France's national research agency, the CNRS. Daniel Guerreau, the institute's deputy scientific director, says that the decision was a simple budgetary one. "Radioactive beams are considered the priority," he says.

But some researchers dispute the idea that existing stable beams are obsolete. They accept that, for most work on exotic nuclei, stable beams will eventually be unable to compete with radioactive-beam technology. But the first radioactive-beam machines, such as the recently opened SPIRAL in France (see Nature 416, 114; 2002), generate beams of limited intensity, and will not fulfil the requirements of some experiments.

Diverse experimental needs mean that even if the next generation of high-intensity machines is built, researchers say that a mixture of machines will still be needed. "Sometimes you have a question where you need a radioactive beam, whereas sometimes you need a stable beam," says Herbert Hübel, a physicist at the University of Bonn.

Moreover, although planned facilities such as the Rare Isotope Accelerator proposed at the Argonne National Laboratory in Illinois, the Eurisol project, and another machine at RIKEN in Japan, would combine many of the best features of existing accelerators, they will not come online for at least eight years.

But Daniel Huss, director of the Institute of

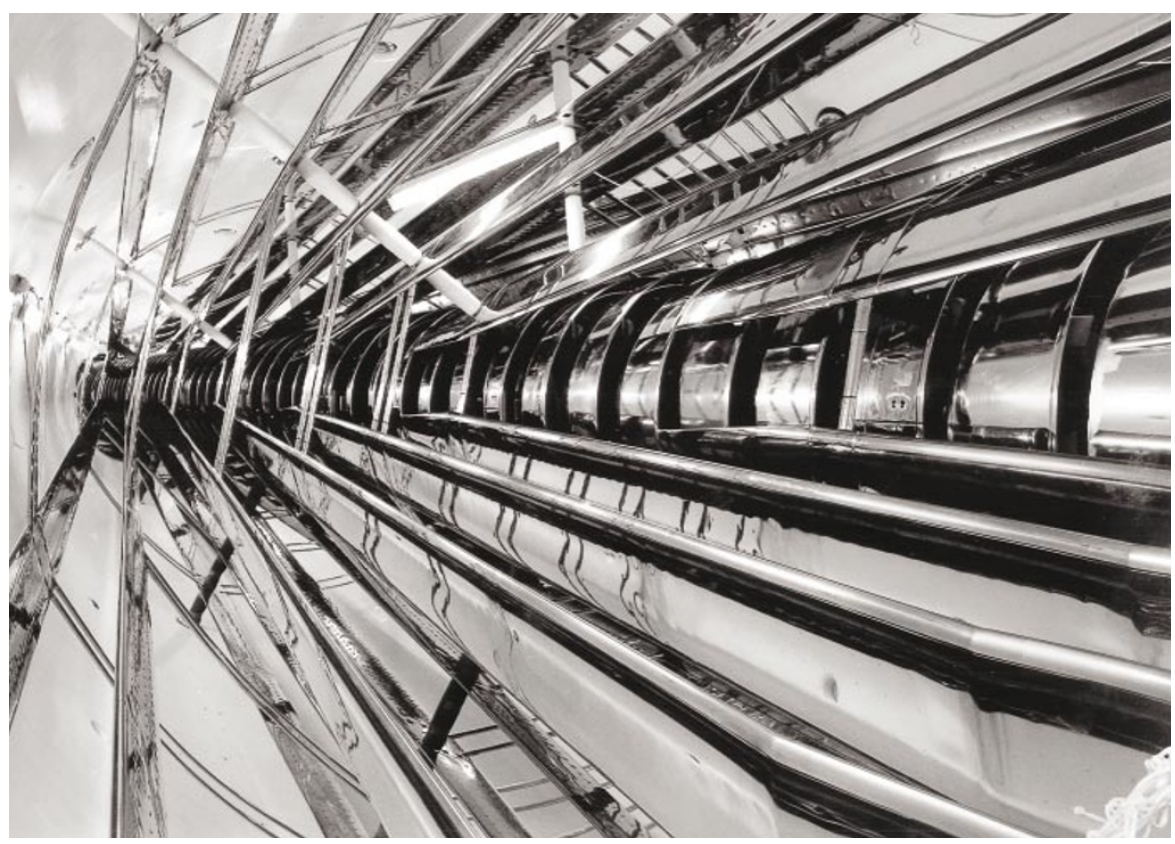

Unstable issue: there is disagreement over whether the Vivitron's stable-beam technology remains useful.

Subatomic Research, which houses the Vivitron, is unmoved by such protests. "I entirely agree with the decision," he says. "The physics we do on Vivitron is no longer a priority."

But the move has come under fire from Juha Äystö, chairman of the Nuclear Physics European Collaboration Committee (NuPECC), which meets under the auspices of the European Science Foundation to produce five-year plans for nuclear physics, similar to those created by the US Nuclear Science Advisory Committee (see below).

Äystö says that researchers cannot simply move elsewhere, as beam time in Europe is already oversubscribed. "There is still very important physics to do on stable beams," he says. NuPECC's next plan is due to be published at the end of this year, and Äystö says that he is "disappointed" that the CNRS has taken its decision without waiting for it.

Several hundred Vivitron users from outside France are now planning to challenge the closure, which they claim will hit students particularly hard. "I have eight $\mathrm{PhD}$ students," one of them complains. "Where are they going to get the data to complete their theses?"

\section{US physicists unite behind big ideas}

\section{Geoff Brumfiel, Washington}

Nuclear physicists in the United States are rallying behind a plan to build two major new facilities in the next 10 years.

The two proposed facilities, for research on rare isotopes and on neutrinos, respectively, are backed in a strategic plan just endorsed by the US government's Nuclear Science Advisory Committee (NSAC). But the committee says that the facilities should not go ahead unless extra money is made available for their construction - and this is cash that even committee members acknowledge will be hard to come by.

The Rare Isotope Accelerator (RIA), the panel's top-priority new facility, would allow nuclear physicists to observe 'exotic' isotopes. The RIA would be more flexible than existing isotope accelerators, its advocates say. Heavy ions would be accelerated into fixed targets at high energies, where their impact would produce a range of rare, short-lived isotopes that could be separated and studied. Researchers think that a better knowledge of such isotopes will further the understanding of how heavy elements form inside supernovae.

The second facility backed by the panel, the National Underground Science Laboratory (NUSL), would study the almost-undetectable particles called neutrinos. The laboratory would be buried some 7 kilometres below ground, to shield its detectors from cosmic rays and other unwanted signals. Currently, US researchers interested in neutrinos must work at underground detector facilities in Japan, Canada or Europe. Neutrino research is part of the US nuclear physics programme for historical reasons. 


\section{University research to touch base with NASA}

Tony Reichhardt, Washington

NASA centres are being told to reach out to universities in a bid to sharpen their research edge.

As a first step, the space agency is seeking a Californian university to conduct multidisciplinary research and engineering under contract to its Ames Research Center, near San Francisco.

The arrangement is expected to form part of a wider drive by NASA to contract out more of its research and engineering to universities and private companies. New NASA administrator Sean O'Keefe, and the White House Office of Management and Budget $(\mathrm{OMB})$, where he used to work, have each made a public priority of "competitive sourcing" of government activities.

NASA is planning to set up a University Affiliated Research Center (UARC) near Ames, a type of arrangement pioneered by the Department of Defense in facilities such as the Applied Physics Laboratory at Johns Hopkins University in Baltimore, Maryland, in which the centre performs specific research tasks under contract to the sponsor.

The planned centre would focus on areas such as information technology, biotechnology, astrobiology and nanotechnology. Located nearby in a still-to-be-developed NASA Research Park, the UARC would work in close collaboration with what the project's planners call a "lean, civil-servicebased core-research centre" at Ames.

NASA hopes that the mix of university faculty, students and NASA researchers at the centre will draw new talent and stimulate

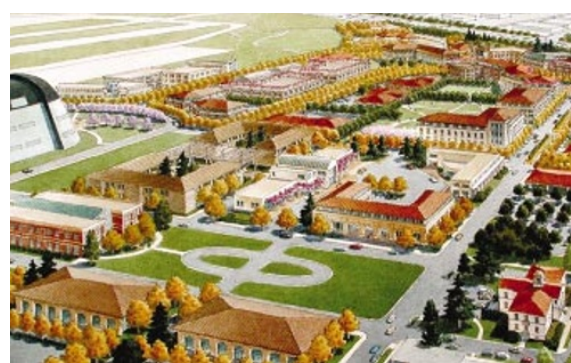

Drawing on expertise: NASA's university-linked centre will be part of its planned research park.

multidisciplinary research and education.

The UARC contract would differ in size and scope from NASA's arrangement with the California Institute of Technology to operate the Jet Propulsion Laboratory (JPL) in Pasadena, California. Whereas the much larger JPL, which has an annual budget of around $\$ 1$ billion, is encouraged to be entrepreneurial and to seek work from sources other than NASA, the UARC would be more tightly bound to its sponsor, and outside work would be limited.

Ames asked for expressions of interest from universities in February. If the idea moves forward as planned, a formal solicitation for a UARC operator would go out in August, with a contract to be awarded next February. The budget for the UARC is estimated at between $\$ 10$ million and \$20 million for the first year, and $\$ 100$ million over a five-year period.

NASA and other federal agencies are under pressure to identify jobs that can be transferred to the private sector. In documents accompanying NASA's 2003 budget request earlier this year, the Bush administration scolded the agency for not going far enough in this regard. The documents identified the Ames UARC as a "pathfinder effort" in outsourcing, and said future efforts "may include consolidating some NASA facilities with military installations."

NASA tried outsourcing some of its inhouse research activities in the mid-1990s, by creating "science institutes" affiliated with universities, but had little success (see Nature 380, 7; 1996). Personnel and management issues have proved particularly thorny whenever the subject of privatizing government research jobs is raised. NASA scientists who transferred last year from the Marshall Space Flight Center in Huntsville, Alabama, to a new National Space Science and Technology Center affiliated with Alabama universities have retained their civilservice status and benefits.

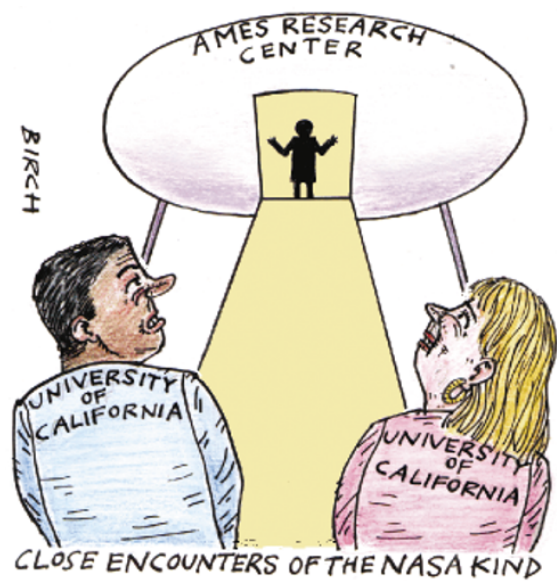

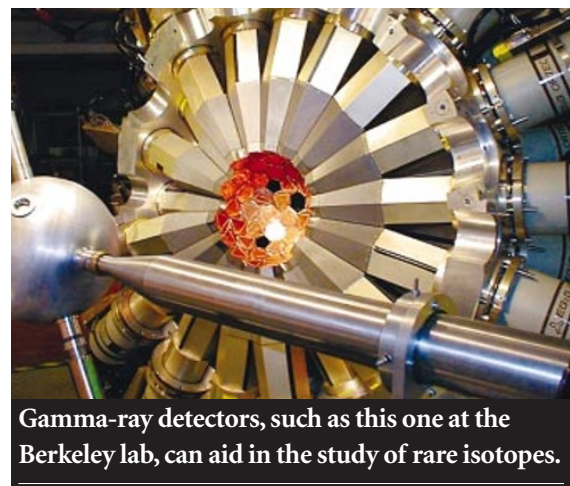

But members of NSAC concede that it will be tough finding money for the two facilities. With its cost estimated at $\$ 700$ million, the RIA would put enormous pressure on the physics budgets of the Department of Energy and the National Science Foundation (NSF).
Even so, says James Symons, chair of the committee and a nuclear physicist at Lawrence Berkeley National Laboratory in California, the country should start thinking about the project now. In the end, Symons thinks, any decision to build the RIA will depend on how the proposal stacks up against desired big projects in other subdisciplines, such as the Next Linear Collider being sought by high-energy physicists.

The NUSL proposal is associated with a site at the Homestake mine in South Dakota (see Nature 415, 105; 2002), and has become an issue in a hotly contested campaign for November's election of a South Dakota senator. But the NSF has responded cautiously to suggestions that it should help to build the NUSL, as this would strain its limited budget for large facilities. "There are great political machinations involved," says
NSAC member Alice Mignerey, a nuclear physicist at the University of Maryland. "But we were very careful that it was the science that drove the recommendation."

Top of the priority list in the 10-year plan, however, is full-capacity operation of the two largest existing nuclear-physics facilities - the Relativistic Heavy Ion Collider at Brookhaven National Laboratory in New York state, and the Continuous Electron Beam Accelerator Facility at the Jefferson Laboratory in Virginia. A proposed $6 \%$ increase in next year's nuclear-physics budget at the Department of Energy would allow that goal to be met immediately, if it is approved by Congress (see Nature 416, 251; 2002). Symons believes that nuclear physicists would then be poised to make some exciting discoveries. "I think this is going to be a great decade for our field," he says. 